\title{
Cerebral venous thrombosis in a patient with mild COVID-19 infection
}

\section{Dear Editor,}

Emerging reports suggest venous and arterial thromboembolic diseases can complicate recovery from COVID-19. Postulated mechanisms include hypercoagulability, hypoxia, immobilisation, excessive inflammation and diffuse intravascular coagulation, especially in patients with severe COVID-19 infection. ${ }^{1-5}$ Nauka et al. ${ }^{6}$ provided vital insight that thrombotic complications can happen in a patient with non-critically ill COVID-19 infection. They reported a case of deep venous thrombosis (DVT) in a patient with mild COVID-19 infection and was treated with apixaban.

We report a case of acute cerebral venous thrombosis (CVT) in a patient with mild COVID-19 infection who was then treated with dabigatran. This case highlights the importance of a high degree of suspicion in diagnosing thromboembolic complications in mild COVID-19 patients. It also raises the question whether some of the headache cases reported with COVID-19 infection may be caused by undiagnosed CVT.

A 35-year-old Bangladeshi man with no past medical history presented with 3 days of fever, cough and headache. He stays in a dormitory at a construction site, with recent clustered outbreak of COVID-19 infections. His headache was described as a continuous pressure located over the neck and occipital area with no associated symptoms of nausea, vomiting, photophobia, phonophobia, or associated focal neurological deficits. The headache did not worsen with straining or by postural change.

His vital signs were normal. Pulse oximetry was $97 \%$ on room air and he did not require oxygen supplementation. Lung examination was normal. Neurological examination was normal with no signs of meningism, and no tenderness over the temporal arteries. Bedside fundoscopy showed no sign of papilloedema. Chest X-ray was performed on admission and was normal. He was admitted to the isolation ward. The SARS-CoV-2 polymerase chain reaction assay from his nasal swab was positive. However, COVID-19 serology test was not performed.

His white blood cell count was $6.9 \times 10^{9} / \mathrm{L}$, with normal neutrophils and lymphocytes counts. His platelet count was $187 \times 10^{9} / \mathrm{L}$. Serum urea was $2.9 \mathrm{mmol} / \mathrm{L}$ and serum creatinine was $81 \mathrm{mmol} / \mathrm{L}$. Coagulation profile was normal: prothrombin time (PT) of 10.1 seconds, activated partial prothrombin time (APTT) of 27.7 seconds, fibrinogen level $2.5 \mathrm{~g} / \mathrm{L}$, d-dimer level $<0.19 \mathrm{mg} / \mathrm{L}$ fibrinogen equivalent units (FEU). Other blood tests such as lactate dehydrogenase (LDH) of 341 units/L, C-reactive protein (CRP) of $0.6 \mathrm{mg} / \mathrm{L}$ and ferritin of $189 \mu \mathrm{g} / \mathrm{L}$ suggested there was no significant systemic inflammation.

He was given paracetamol and benzydamine hydrochloride lozenges for fever and sore throat, respectively. No thromboprophylaxis was initiated. He was categorised with mild infection based on World Health Organization interim guidance. ${ }^{9}$

His headache was treated with etoricoxib but continued to worsen over the next 3 days. A brain magnetic resonance imaging (MRI) was performed and showed no features of acute encephalitis/meningitis, acute infarction or haemorrhage. Brain magnetic resonance venogram showed loss of normal T2 flow void in the left transverse, and sigmoid sinuses with filling defects suggested CVT (Figs. 1a and 1b). A lumbar puncture was performed with an opening pressure of $18.5 \mathrm{~cm}$ $\mathrm{H}_{2} \mathrm{O}$, and closing pressure of $16.8 \mathrm{~cm} \mathrm{H}_{2} \mathrm{O}$. Cerebral spinal fluid examination showed normal white cell count of 3 cells $/ \mathrm{mm}^{3}$, red blood cell counts of 1,000 cells $/ \mathrm{mm}^{3}$ due to a traumatic tap and protein of $0.76 \mathrm{mg} / \mathrm{dL}$.

Anticoagulation with oral dabigatran $150 \mathrm{mg}$ BD was initiated. He was screened for other thromboembolic manifestations and no asymptomatic DVT was found. There was no family history of venous thrombosis upon further history taking. His headache improved and there were no new neurological symptoms or signs. Computed tomography venogram 1 month later showed resolution of CVT (Fig. 1c). He was planned for 3 months of dabigatran treatment.

Interestingly, there were no signs of hypercoagulability (with normal d-dimer and serum fibrinogen), dehydration, hypoxia or diffuse intravascular coagulation in this case, contrary to most reported cases of venous thromboembolism in COVID-19 patients. ${ }^{1,7}$ This raises the importance of awareness to consider CVT in a COVID-19 patient without any signs of hypercoagulability and presenting with subtle symptoms such as headache.

Headache is the second most common neurological symptom reported in COVID-19 patients, reported to have been experienced by $13.1 \%$ of patients. ${ }^{8}$ As many may not have been investigated for their headaches, it is unknown how many have had undiagnosed CVT. With 

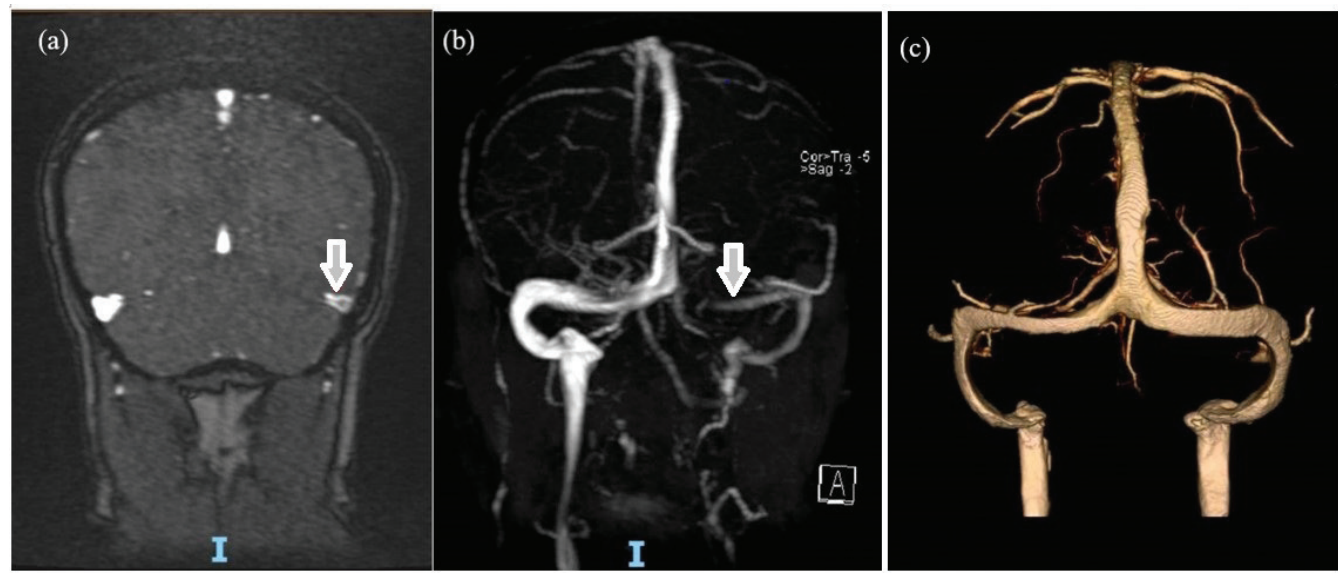

Fig. 1. Brain radiological findings. Central filling defects (see 2 arrows) seen in (a) left transverse sinus on T1 post-contrast MRI brain and (b) magnetic resonance venogram, showing left transverse sinus and sigmoid sinus thrombosis. (c) CTV showed resolution of sinus thrombosis.

CTV: computed tomography venogram; MRI: magnetic resonance imaging

the known association of hypercoagulability with COVID-19 infection, headache should be evaluated thoroughly with a detailed history and examination, and imaging including venography should be considered.

Incidence of thrombotic complications in intensive care unit COVID-19 patients was reported as $31 \%$, with $27 \%$ of venous and $3.7 \%$ of arterial thrombotic events. ${ }^{1}$ Thromboprophylaxis is recommended to be given to COVID-19 patients, ${ }^{10}$ which may help to prevent the development of CVT. In addition, good hydration should be maintained.

CVT associated with COVID-19 infection has been reported worldwide. ${ }^{11-14}$ In these reports, the patients with CVT had abnormal coagulation profile such as elevated d-dimer, ${ }^{12,13}$ low platelet ${ }^{13}$ and high fibrinogen ${ }^{11}$ or suggestions of systemic inflammation such as high $\mathrm{CRP}^{11,12}$ and high $\mathrm{LDH} .{ }^{12}$ This case is unique as the patient's coagulation profile (platelet count, d-dimer, PT, APTT) and inflammatory makers (CRP, LDH, ferritin) were normal. Kow et al. ${ }^{15}$ suggested that low molecular weight heparin may be worth considering in treating COVID-19 patients with CVT due to better safety and efficacy. To our best knowledge, this is the first CVT in COVID-19 infection treated successfully with dabigatran as shown by the resolution of CVT in the computed tomography venogram.

The limitation of this case was that thrombophilia screen was not performed during admission as the processing institute did not accept the patient's blood samples, based on institutional protocol in view of positive COVID-19 infection. Outpatient follow-up plan spanned 3 months after admission, for review and thrombophilia screen. Unfortunately, he did not attend the planned follow-up.

In conclusion, this case highlights the importance for awareness of thrombotic complications in mild COVID-19 cases. In addition, further research is required to study thromboprophylaxis for patients with COVID-19.

\section{REFERENCES}

1. Klok FA, Kruip MJHA, van der Meer NJM, et al. Incidence of thrombotic complications in critically ill ICU patients with COVID-19. Thromb Res 2020;191:145-7.

2. Chen $\mathrm{T}, \mathrm{Wu} \mathrm{D}$, Chen $\mathrm{H}$, et al. Clinical characteristics of 113 deceased patients with coronavirus disease 2019: retrospective study. BMJ 2020;368:m1091.

3. Guan WJ, Ni ZY, Hu Y, et al. Clinical characteristics of coronavirus disease 2019 in China. N Engl J Med 2020;382:1708-20.

4. Wang $\mathrm{D}, \mathrm{Hu} \mathrm{B}, \mathrm{Hu} \mathrm{C}$, et al. Clinical characteristics of 138 Hospitalized Patients with 2019 Novel Coronavirus-Infected Pneumonia in Wuhan, China. JAMA 2020;323:1061-9.

5. Zhou F, Yu T, Du R, et al. Clinical course and risk factors for mortality of adult inpatients with COVID-19 in Wuhan, China: a retrospective cohort study. Lancet 2020;395:1054-62.

6. Nauka PC, Oran E, Chekuri S, et al. Deep venous thrombosis in a non-critically ill patient with novel COVID-19 infection. Thromb Res 2020;192:27-8.

7. Zhou F, Yu T, Du R, et al. Clinical course and risk factors for mortality of adult inpatients with COVID-19 in Wuhan, China: a retrospective cohort study. Lancet 2020;395:1054-62.

8. Mao L, Jin H, Wang M, et al. Neurologic Manifestations of Hospitalized Patients with Coronavirus Disease 2019 in Wuhan, China. JAMA Neurol 2020;77:683-90.

9. World Health Organization. Clinical management of severe acute respiratory infection (SARI) when COVID-19 disease is suspected. Interim guidance. 27 May 2020. Available at: https://www.who.int/ 
publications/i/item/clinical-management-of-covid-19. Accessed on 8 Oct 2020.

10. Kollias A, Kyriakoulis KG, Dimakakos E, et al. Thromboembolic risk and anticoagulant therapy in COVID-19 patients: emerging evidence and call for action. Br J Haematol 2020;189:846-7.

11. Hughes $C$, Nichols $T$, Pike $M$, et al. Cerebral venous sinus thrombosis as a presentation of COVID-19. Eur J Case Rep Intern Med 2020;7:00169

12. Klein DE, Libman R, Kirsch C, et al. Cerebral venous thrombosis: A typical presentation of COVID-19 in the young. J Stroke Cerebrovasc Dis 2020;29:104989.

13. Garaci F, Di Giuliano F, Picchi E, et al. Venous cerebral thrombosis in COVID-19 patient. J Neurol Sci 2020;414:116871.

14. Cavalcanti DD, Raz E, Shapiro M, et al. Cerebral Venous Thrombosis Associated with COVID-19. AJNR Am J Neuroradiol 2020;41:1370-6.

15. Kow CS, Zaihan AF, Hasan SS. Anticoagulant approach in COVID-19 patients with cerebral venous thrombosis. J Stroke Cerebrovasc Dis 2020:105222.
Yu Zhi Pang, ${ }^{1} M D$, Humaira Shafi, ${ }^{2} M B B S$, Zheng Cong Lee, ${ }^{2}{ }_{M D}$, Simon Kang Seng Ting, ${ }^{3} M B B S$, Deidre Anne De Silva, ${ }^{4}{ }_{M B B S}$

${ }^{1}$ Division of Neurology, Department of General Medicine, Changi General Hospital, Singapore

${ }^{2}$ Department of Infectious Disease, Changi General Hospital, Singapore

${ }^{3}$ Department of Neurology, National Neuroscience Institute, Changi General Hospital Campus, Singapore

${ }^{4}$ Department of Neurology, National Neuroscience Institute, Singapore General Hospital Campus, Singapore

Correspondence: Dr Yu Zhi Pang, Division of Neurology, Department of General Medicine, Changi General Hospital, 2 Simei Street 3, Singapore 529889 .

Email: pang.yu.zhi@singhealth.com.sg 